\title{
Effective Service Capacity Analysis of Opportunistic Multi-Carrier OFDMA Systems
}

\author{
James Gross, Marco Weyres \\ Mobile Network Performance Group \\ RWTH Aachen University \\ Aachen, Germany \\ gross|weyres@umic.rwth-aachen.de
}

\author{
Adam Wolisz \\ Telecommunication Networks Group \\ Technische Universität Berlin \\ Berlin, Germany \\ wolisz@tkn.tu-berlin.de
}

\begin{abstract}
Exact queuing-theoretic modeling of wireless systems is tough due to the complex service processes that arise from the interaction of the wireless channel with state-of-the-art signal processing algorithms. Nevertheless, with the rise of video applications like streaming and telephony in upcoming fourth generation cellular networks, such analysis is important for devising admission control strategies. In this paper we apply recent insights into approximation approaches for queuing systems to opportunistic OFDMA systems and study different approaches for applying the modulation type to OFDM subcarriers. In particular, we provide analytical closed-form expressions for the delay distribution of three opportunistically scheduling OFDMA systems (adaptive modulation, link adaptation per terminal, link adaptation over all subcarriers). After validating these expressions, we evaluate the different system designs numerically. We find that although adaptive modulation systems have a higher complexity, their queuing performance is only slightly superior to systems with link adaptation per terminal. Furthermore, the systems feature in general an optimal number of terminals that can be supported in the cell, leveraging multi-user diversity with limited available resources. Nevertheless, these optima change from scheme to scheme.
\end{abstract}

\section{Categories and Subject Descriptors}

G.3 [Probability and Statistics]: Queuing Theory

\section{Keywords}

QoS, Effective Capacity

\section{INTRODUCTION}

Queuing-theoretic means are without doubt one of the major tools for performance analysis of network protocols and

\footnotetext{
* This work was funded by the German Research Foundation (DFG) under grant 'Schedulability of Cognitive OFDMA Systems'.
}

Permission to make digital or hard copies of all or part of this work for personal or classroom use is granted without fee provided that copies are not made or distributed for profit or commercial advantage and that copies bear this notice and the full citation on the first page. To copy otherwise, to republish, to post on servers or to redistribute to lists, requires prior specific permission and/or a fee.

Q2SWinet'12, October 24-25, 2012, Paphos, Cyprus.

Copyright 2012 ACM 978-1-4503-1619-4/12/10 ...\$15.00. algorithms [8]. In such an approach, a networking system is modeled by a server with a (finite) storage space where service requests arrive randomly to the storage space and are served by the network, potentially requiring a random service time. From such a starting point one typically first studies the stability of the queue. If this can be established, more complex metrics are investigated such as average queue length, average queuing delay but also distributions of the queuing delay and length (among many other metrics). The advantage of such an analytical approach is clear: It is a perfect fit for the requirements of delay-sensitive applications where - for example - the queuing delay distribution allows immediately to judge the quality-of-service (QoS) provided by the network. Hence, the analysis is applicable in admission control but also for network dimensioning and protocol development. On the other hand, exact queuing-theoretic models can only be found for a handful of real systems. The mathematical problem to overcome here lies in the interaction between (random) arrivals and service and their probabilistic impact on the state space of the queuing system. Often, this limits precise analysis such that only average metrics can be considered exactly.

This is especially true for wireless systems. As the service process of wireless systems is always the result of the interaction between the (random) wireless channel variations and complex signal processing algorithms at transmitter and receiver, to date we do not have exact queuing models for wireless systems in general. In addition, as the development of wireless systems is predominantly driven by the physical layer community, standard performance metrics are rather the (average) bit error rate or maximal throughput (ergodic capacity) of the system instead of queuing-related metrics. Nevertheless, there is a recognized need for analytical understanding of the impact of the wireless physical layer on metrics of the application layer, especially with respect to the queuing delay distribution (as it is a primary performance metric for delay-sensitive flows such as voice over IP and video streaming/telephony). This is due to the expectation that especially video telephony will become a major application within this decade.

As exact queuing analysis of wireless systems turns out to be quite difficult to achieve, one can instead apply approximation techniques. One successful technique for such an approximation consists of utilizing the Chernov bound of the steady-state queue length of the system and deriving from this starting point exponential bounds for the steady state queue length distribution. Originally, this approach was applied to bound the queue length of a fixed-rate multiplexing 
link to which a set of data sources with random rates (voice calls) is connected [2]. However, the same technique can be 'turned around' to bound the queue length distribution of a system given a deterministic (constant) arrival rate and a random service process. This technique has been applied to several wireless systems in a successful way (see Section 2.4 for a detailed discussion) and was originally introduced by $\mathrm{Wu}$ et al. in [15]. It is known under the term 'effective capacity' which refers to a rather complex expression of the random service process. Nevertheless, it is a suitable analytical approach for determining queuing performance of wireless systems and devising on top of this admission control strategies, for example. Note that any result for the queue length can be converted to statements regarding the queuing delay.

In this work, we extend this line of research to an important class of wireless multi-user systems. In particular, we focus on multi-carrier systems and consider the effective service capacity of various different design alternatives. This is mainly motivated by the need for analytical models for admission control of delay-sensitive applications such as voice and video applications in upcoming cellular networks. In such networks the central component that determines the systems performance is the resource scheduler at the base station. In upcoming OFDM-based networks like 3GPP LTE [3] this scheduler exploits instantaneous channel state information and assigns OFDM subcarriers dynamically to terminals from frame to frame [1]. Typical assignment policies are either opportunistic scheduling or variants of proportional-fair scheduling. However, the obtained subsets of subcarriers can be utilized in different ways to forward data to the terminals in the down-link. We consider in this paper an opportunistic resource scheduler and distinguish three different cases for adapting to the channel states: (1) Adaptive modulation where the modulation type can be set individually per subcarrier; (2) Link adaptation per terminal where the modulation type is set equal over all subcarriers assigned currently to the same terminal; (3) Link adaptation over all terminals where all subcarriers of the system are utilized with the same modulation type regardless of which terminal the subcarriers are assigned to.

Clearly, adaptive modulation is associated with a higher complexity in terms of transceiver complexity and signaling overhead. This is significantly lower for link adaptation systems. However, as the performance of link adaptation is mainly driven by the subcarriers with the worst channel states, there is potentially also a significant performance loss associated to them. We are especially interested in the performance difference of these systems in terms of link layer queuing metrics. Our contributions are twofold. We first provide and validate mathematical expressions for the queuing performance of the systems based on the effective service capacity framework. We then provide a numerical analysis demonstrating (surprisingly) that the performance difference between opportunistic scheduling with adaptive modulation and link adaptation per terminal is negligible whereas link adaptation over all terminals provides a significantly lower performance. This is interesting as the underlying assumptions in this study regarding the link adaptation systems are rather pessimistic.

The remaining paper is structured in the following way: First, we introduce the system model and the applied framework of the effective service capacity in Section 2. In Section
3 we derive then the queuing performance of the different system approaches. Section 4 contains the validation of our analytical work by simulations as well as a numerical evaluation. We finally conclude the paper in Section 5.

\section{PRELIMINARIES}

In the following we first present the system model and the problem statement. Section 2.3 gives a brief overview of the effective service capacity framework, while Section 2.4 summarizes related work.

\subsection{System Model}

We consider a multi-user communication setting where an access point coordinates all data transmissions. There are $J$ wireless terminals in the cell. Time is divided into frames of duration $T_{\mathrm{f}}$. The system applies OFDM as transmission scheme and utilizes a bandwidth of $B[\mathrm{~Hz}]$ which is split into $N$ subcarriers. Each subcarrier features a symbol duration of $T_{\mathcal{S}}$ (including the guard period) where $\mathcal{S}=T_{\mathrm{f}} / T_{\mathcal{S}}$ denotes the number of symbols per frame. For down-link data transmission the access point can apply a maximum transmit power of $P_{\mathrm{dl}}[\mathrm{W}]$ per subcarrier.

Each terminal receives a constant stream of data which is buffered at the access point upon transmission. Each flow arriving for terminal $j$ has a rate of $r_{j}$ bits per frame duration $T_{\mathrm{f}}$. All data flows are subject to quality-of-service requirements given by the pair $\left\{d_{j}, \mathcal{P}_{j}\right\}$ where $d_{j}$ stands for a maximum tolerable delay of each bit of data flow $j$ and $\mathcal{P}_{j}$ denotes the corresponding outage probability, i.e. the probability that the delay target is not met for a given bit of the flow $j$. Denote the cumulative arrival process of flow $j$ to the link layer at the access point up to frame $i$ by $A_{j}[i]=\sum_{1}^{i} a_{j}[i]=i \cdot r_{j}$ and $S_{j}[i]=\sum_{1}^{i} s_{j}[i]$ the cumulative service process respectively.

Let us focus on frame $i$ in the following. Prior to the upcoming transmission slot, the access point receives feed-back regarding all current channel states of all subcarriers to all terminals. In general, the channel state per subcarrier is a random variable. It is characterized by the instantaneous SNR $\gamma_{j, n}=P_{\mathrm{dl}} \cdot h_{j, n}^{2} /\left(\sigma^{2}\right)$ where $h_{j, n}$ is the instantaneous (random) channel magnitude of subcarrier $n$ to terminal $j$ and $\sigma^{2}$ models the noise power per subcarrier. We assume the channel magnitude $h_{j, n}$ to be Rayleigh-distributed and hence the instantaneous SNR $\gamma_{j, n}$ to be exponentially distributed with mean $\bar{\gamma}_{j, n}$. The channel state $\gamma_{j, n}$ is furthermore assumed to be statistically independent in time and in frequency. In addition, the channel states are also spatially independent such that $\gamma_{j, n}$ and $\gamma_{i, n}$ are independent random variables. Finally, we assume in the following that due to slowly-varying power control at the access point the average SNRs are kept equal, i.e. $\bar{\gamma}_{j, n}$ is equal for all $J$ terminals and all $N$ subcarriers (power control can react to the effect of shadowing but not to the effect of time- and frequency-selective fading). Denote this average SNR by $\bar{\gamma}$.

Depending on the channel states and the system set-up, different transmission characteristics can be exploited to convey the queued data at the access point. In particular, we consider three different system set-ups that differ in the way the subcarriers are modulated, but use the same multiple access scheme. In all three set-ups we assume terminals to be served in an OFDMA fashion. More precisely, for each frame subcarriers are assigned opportunistically and are thus 
assigned to the terminal which has the best SNR for the corresponding subcarrier. To accomplish this the access point is assumed to have the required channel state information to perform such assignments. However, given the subcarrier assignments the three considered systems apply the modulation types differently:

- OFDMA with Adaptive Modulation: In the first system set-up we assume that per subcarrier the modulation type can be varied individually. Hence, if subcarrier $n$ is assigned to terminal $j$ and has a current SNR of $\gamma_{j, n}$ then $\mathcal{S} \cdot \log _{2}\left(1+\gamma_{j, n}\right)$ bits can be conveyed over this subcarrier for the upcoming time slot. The total service $s_{j}$ received during the slot is then simply the sum of the service over all assigned subcarriers. Obviously, this scheme is complex to realize but will also lead to a fine-grained channel adaptation.

- OFDMA with Link Adaptation: For the second system set-up we assume that over all subcarriers only one modulation type can be applied (regardless of the assignment of the subcarriers to terminals). In such a link adaptation system an important question relates to the selection of the modulation type for all subcarriers (as the different subcarriers have a strongly varying SNR). Here the notion of the effective SNR has been established (especially in the context of LTE systems). Given $N$ subcarriers with varying SNRs the effective SNR is in the range between $\gamma_{\text {min }} \leq \gamma_{\text {eff }} \leq$ $\gamma_{\min }+\beta \cdot \log (N)$ where $\beta$ is a constant [12] and $\gamma_{\min }$ is the lowest SNR among the different SNRs of all subcarriers. Due to this, we choose a performance model where the rate depends on the lowest SNR among all subcarriers. Hence, the rate that can be achieved over $N$ subcarriers is given by $N \cdot \mathcal{S} \cdot \log _{2}\left(1+\gamma_{\min }\right)$, even if the other subcarriers are in a much better SNR state than $\gamma_{\min }$.

- OFDMA with Link Adaptation per Terminal: In the last system set-up for the modulation we also follow the link adaptation approach. However, the link adaptation is applied per terminal. Therefore, subcarriers assigned to different terminals might be utilized with a different modulation scheme while all subcarriers assigned to one terminal are set to the same modulation type. Accordingly, the modulation per subcarrier assignment set is chosen depending on the subcarrier with the lowest SNR among all assigned subcarrier for each specific terminal. In general, this system approach represents the LTE base design [3] and can be seen as a compromise between employing adaptive modulation per subcarrier on the one hand and employing link adaptation over all subcarriers (regardless of the terminal assignments).

The first two set-ups result clearly in a different stochastic behavior of the service process, whereas the third case is a generalization of the second one.

\subsection{Problem Statement}

In this work we are interested in the impact of the three different system set-ups on the resulting queuing performance. The systems bring clearly a different trade-off in terms of performance and complexity. For instance, adaptive modulation requires a more complex transceiver struc- ture. In addition, this variant requires the existence of a high-rate signaling channel to indicate the used subcarrier/modulation combinations. We are interested in the resulting performance trade-off of these various systems with their different service characteristics and implementation complexities: How big is the performance gain that stems from OFDMA channel access and adaptive modulation for different QoS requirements? How much better gets the queuing performance theoretically if adaptive modulation would be applied instead of link adaptation? The novelty of our work stems from an analytical consideration of QoS metrics by deriving the effective service capacities of the corresponding systems and the validation of these derivations. In the following, we give a brief overview of the effective service capacity framework.

\subsection{Queue Performance Approximation by Effective Service Capacity Framework}

The mathematical framework from which the effective service capacity is obtained allows to approximate the distribution of the steady-state queue length of a stable queuing system. It is therefore a tool for analysis of arbitrary service processes in a queuing system. The framework was originally applied to characterize the queue length for arbitrary arrival processes (source flows) which are served in a queuing system by a constant rate. In this context, the so called effective bandwidth of the arrival process needs to be derived to bound the queue length distribution [2]. In the following we give a brief introduction to the framework with respect to effective service capacity. Based on the notation introduced above, we can express the random queue length regarding terminal $j$ at time $i$ by:

$$
Q_{j}[i]=\max _{0 \leq k \leq i}\left((i-k) \cdot r_{j}-\left(S_{j}[i]-S_{j}[k]\right)\right) .
$$

Let us consider that the arrival and service process are stationary. Furthermore, assume that the queue is stable as the average service rate is larger than the average arrival rate. Hence, the random queue length $Q_{j}[i]$ at frame $i$ converges to the steady-state random queue length $Q_{j}$. We are interested in characterizing the long-term statistics $\operatorname{Pr} .\left\{Q_{j}\right\}$ of the queue length. The framework of effective service capacity gives us the following upper bound:

$$
\operatorname{Pr} .\left\{Q_{j}>x\right\} \approx K \cdot e^{-\theta \cdot x},
$$

where $K$ is the probability that the queue is non-empty and $\theta$ is the so called quality-of-service exponent. Clearly, the bigger the QoS exponent $\theta$ is, the better is the bound for the backlog probability. An upper bound for $\theta$ can be found from the constraint:

$$
r_{j}<-\frac{\Lambda(-\theta)}{\theta}
$$

where $\Lambda(\theta)$ is called the log-moment generating function of the increments of the cumulative service process $S_{j}[i]$ defined as (assuming the increments to be stationary as well):

$$
\Lambda(\theta)=\lim _{i \rightarrow \infty} \frac{1}{i} \log \mathrm{E}\left[e^{\theta \cdot\left(S_{j}[i]-S_{j}[0]\right)}\right] .
$$

Finally, the ratio $-\Lambda(-\theta) / \theta$ is referred to as effective service capacity, as the exponential decay of the queue length distribution in Equation (2) is only witnessed if the ratio $-\Lambda(-\theta) / \theta$ is bigger than the constant arrival rate $r_{j}$ of the source. For approximating the queue length distribution, 
the tightest results are obtained from choosing the biggest $\theta$ such that Equation (3) still holds.

So far we have only considered the random queue length. Corresponding to the notation above, we denote by $D_{j}[i]$ the random queuing delay of the head-of-line bit during frame $i$ for terminal $j$. This converges in the long-run to the random steady state queuing delay $D_{j}$ of the head-of-line bit. As the arrival process has a fixed rate, the steady-state queue length statistics are related to the steady-state delay statistics of the head-of-line bit. Hence, a queue length of $Q_{j}=q$ is associated with a current delay of the head-of-line bit of $D_{j}=q / r_{j}$. This yields the following approximation for the steady-state delay distribution from Equation (2):

$$
\operatorname{Pr} .\left\{D_{j}>d\right\} \approx K \cdot e^{-\theta^{*} \cdot r_{j} \cdot d} .
$$

A considerable challenge in determining the effective service capacity is the characterization of the log-moment generating function. If the increments $s_{j}[i]$ of the cumulative service process $S_{j}[i]$ can be assumed to be i.i.d., a convenient simplification is to obtain the log-moment generating function via the law of the large numbers [14]. Denoting the increments in the following simply by $s_{j}$, the effective service capacity can be obtained by:

$$
\begin{aligned}
-\frac{\Lambda(-\theta)}{\theta} & =\lim _{i \rightarrow \infty} \frac{1}{i \cdot \theta} \log \mathrm{E}\left[e^{-\theta \cdot s_{j}[i]}\right] \\
& =\mathrm{E}\left[s_{j}[i]\right]-\frac{\theta}{2} \operatorname{Var}\left[s_{j}[i]\right] .
\end{aligned}
$$

It is therefore sufficient to determine the average and the variance of the instantaneous service process $s_{j}$.

Equations (2) and (5) allow to approximate the resulting backlog and queuing delay probability given an arrival process with rate $r_{j}$ and a service process for which the effective service capacity can be derived. In contrast, for admission control we are more interested in the opposite: For a given QoS target (i.e. delay $d_{j}$ and maximum tolerable probability $\mathcal{P}_{j}$ that this delay target can not be achieved) what is the maximum sustainable arrival rate $r_{j}^{*}$ for which these targets can still be met given a certain service process. For this case, we obtain from Equation (5) the following approximation for the maximum sustainable rate (upper bounding $K$ by 1$)$ :

$$
\frac{-\ln \left(\mathcal{P}_{j}\right)+\ln (K)}{d_{j}} \geq r_{j}^{*} \cdot \theta \Rightarrow r_{j}^{*} \cdot \theta \approx-\frac{\ln \left(\mathcal{P}_{j}\right)}{d_{j}} .
$$

This approximation still depends on the QoS exponent $\theta$. From Equation (3) we can obtain an upper limit on the QoS exponent given as $\theta_{\text {max }}$ based on substituting the expression for the effective service capacity from Equation (7). This yields:

$$
\theta_{\max }=2 \cdot \frac{\mathrm{E}\left[s_{j}\right]-r_{j}}{\operatorname{Var}\left[s_{j}\right]}
$$

Using this expression for $\theta$, we can substitute it in Equation (8) to obtain the following relationship for the maximum sustainable arrival rate $r_{j}^{*}$ which has been first proposed by Soret et al. [14]:

$$
\begin{gathered}
2 \cdot\left(\mathrm{E}\left[s_{j}\right] \cdot r_{j}^{*}-\left(r_{j}^{*}\right)^{2}\right) \approx-\frac{\ln \left(\mathcal{P}_{j}\right)}{d_{j}} \cdot \operatorname{Var}\left[s_{j}\right] \Rightarrow \\
r_{j}^{*} \approx \frac{1}{2} \cdot\left(\mathrm{E}\left[s_{j}\right]+\sqrt{\left(\mathrm{E}\left[s_{j}\right]\right)^{2}+\frac{2 \cdot \ln \left(\mathcal{P}_{j}\right)}{d_{j}} \cdot \operatorname{Var}\left[s_{j}\right]}\right)
\end{gathered}
$$

In the following, we use this equation to determine the maximum sustainable source rate. The major difficulty that we will face is obtaining the mean and variance of the instantaneous service process depending on the different system set-ups described in the previous section.

\subsection{Related Work}

The notion of the effective service capacity has been introduced by $\mathrm{Wu}$ in [15]. This original work considered a single wireless fading channel with perfect channel state information at the transmitter and the possibility to adapt to the channel state on a per-symbol base. Difficulties in characterizing the log-moment generating function for a correlated wireless channel forced the authors to consider special cases like low-SNR-regime etc. The work was extended afterwards by numerous further contributions of $\mathrm{Wu}$ et al. for example considering point-to-point communication over frequency-selective fading channels [17], multi-user communication over a single flat-fading channel [18], as well as multi-user communications over parallel, down-link fading channels [16]. Further contributions extending the effective service capacity to other wireless system scenarios have been presented in [7] (extension to point-to-point communication via adaptive resource allocation in multi-carrier systems) or [14] (extension to point-to-point communication of variable rate sources over a single, correlated fading channel). In [13] Soret et al. extended their work to a multiuser case for uncorrelated Rayleigh-fading channels. They investigated the influence of a round robin scheduler and an opportunistic best channel scheduler. However, for both schedulers they only considered single-carrier systems. Also, no link adaptation was investigated. A somewhat related work can be found in [11] where the effective service capacity for multiple users with different scheduling strategies was considered in order to evaluate the benefits of multiuser scheduling. Here the focus was on different schedulers that also take the queue state of the different users into account. However, the author only considered single-carrier systems. Finally, in [6] the author presents a model for considering losses from overestimating the channel state in face of imperfect channel state information. Based on this model, the effective service capacity is derived and the impact of different scheduling strategies is studied. Again, the analysis only applies to a single-carrier system where a single user is served.

In the context of multi-carrier systems, only a few works have been dealing with the resulting impact of resource allocation on queuing metrics. In [10] the authors study the impact of dynamic subcarrier scheduling on link performance. However, they do not have an analytical model for the service process resulting from dynamic scheduling. Instead, they utilize results from simulations. A corresponding analytical model for the service process in OFDMA systems with dynamic resource allocation is introduced in [5] and applied to study the marginal blocking probability of an OFDMA cell. This work has been extended to interferencelimited systems in [9].

\section{ANALYSIS OF THE EFFECTIVE CAP- ACITIES}

In this section we derive the corresponding mean and second moments of the service processes of the different system set-ups. Recall that all system set-ups are considered to 
have perfect channel state information. Furthermore, we assume all subcarrier states to be independent in time, frequency and space while the SNR is exponentially distributed with mean $\bar{\gamma}$ which is the same for all terminals and subcarriers. Finally, given a subcarrier SNR of $\gamma$, the amount of bits that can be transmitted during one frame is given by $\mathcal{S} \cdot \log _{2}(1+\gamma)$ for adaptive modulation whereas for link adaptation the corresponding rate depends on the lowest SNR of all subcarriers in the respective assignment set. Finally, all system set-ups assume an opportunistic resource scheduler at the base station.

\subsection{Effective Capacity of OFDMA with Adap- tive Modulation}

Due to opportunistic scheduling each subcarrier is allocated to the terminal which has the best SNR. Utilizing order statistics [5], this allows us to determine the probability distribution for the SNR of these chosen subcarrier/terminal pairs. Denote the best SNR out of a set of $J$ independent and identically distributed random variables by $\gamma^{(J / J)}$. Then, the resulting density function is given by:

$$
\begin{aligned}
f_{(J / J)}(x) & =J \cdot F_{\gamma_{j}}^{J-1}(x) \cdot f_{\gamma_{j}}(x) \\
& =\frac{J}{\bar{\gamma}} \cdot \sum_{i=0}^{J-1}\left(\begin{array}{c}
J-1 \\
i
\end{array}\right) \cdot(-1)^{i} \cdot e^{-\frac{x \cdot(i+1)}{\bar{\gamma}}} .
\end{aligned}
$$

In order to compute the mean and variance of the service process $s_{j}$, we first notice that each terminal is likely to receive some service in every frame. The exact number of subcarriers that some terminal $j$ will receive for the next frame is a random variable $X_{j}$. Under our assumptions of equal average channel gains $\bar{\gamma}$ for all terminals, $X_{j}$ becomes a binomially distributed random variable with basic probability $1 / J$. Notice that the $X_{j}$ assigned subcarriers all have the same probability density distribution described by (11). This allows us to compute the mean of the service process $s_{j}$ as follows:

$$
\begin{aligned}
\mathrm{E}\left[s_{j}\right]= & \sum_{k=1}^{N} \operatorname{Pr} .\left\{X_{j}=k\right\} \cdot k \cdot \mathrm{E}\left[s_{j}^{(J / J)}\right] \\
= & \frac{N}{J} \cdot \mathcal{S} \int_{0}^{\infty} \log _{2}(1+x) \cdot f_{(J / J)}(x) d x \\
= & \frac{N}{J} \cdot \sum_{i=0}^{J-1}\left(\begin{array}{c}
J-1 \\
i
\end{array}\right) \cdot(-1)^{i} \\
& \cdot \int_{0}^{\infty} \frac{\mathcal{S} \cdot J}{\bar{\gamma}} \cdot \log _{2}(1+x) \cdot e^{-\frac{x \cdot(i+1)}{\bar{\gamma}}} d x \\
= & N \cdot \sum_{i=0}^{J-1}\left(\begin{array}{c}
J-1 \\
i
\end{array}\right) \cdot(-1)^{i} \\
& \cdot \frac{\mathcal{S}}{(i+1) \cdot \ln (2)} \cdot e^{\frac{i+1}{\bar{\gamma}}} \cdot \mathrm{E}_{1}\left(\frac{i+1}{\bar{\gamma}}\right) .
\end{aligned}
$$

For the second moment we obtain:

$$
\begin{aligned}
\mathrm{E}\left[s_{j}^{2}\right] & =\sum_{k=1}^{N} \operatorname{Pr} .\left\{X_{j}=k\right\} \cdot \mathrm{E}\left[\left(s_{j}^{(J / J), 1}+\cdots+s_{j}^{(J / J), k}\right)^{2}\right] \\
& =\sum_{k=1}^{N} \operatorname{Pr} .\left\{X_{j}=k\right\}
\end{aligned}
$$

$$
\begin{aligned}
& \cdot\left(k \cdot \mathrm{E}\left[\left(s_{j}^{(J / J)}\right)^{2}\right]+\left(k^{2}-k\right) \cdot \mathrm{E}^{2}\left[s_{j}^{(J / J)}\right]\right) \\
& =\sum_{k=1}^{N} \operatorname{Pr} \cdot\left\{X_{j}=k\right\} \cdot k \cdot \mathcal{S}^{2} \\
& \int_{0}^{\infty}\left(\log _{2}(1+x)\right)^{2} \cdot f_{(J / J)}(x) d x \\
& +\sum_{k=1}^{N} \operatorname{Pr} .\left\{X_{j}=k\right\} \cdot\left(k^{2}-k\right) \\
& \cdot\left(\mathcal{S} \cdot \int_{0}^{\infty} \log _{2}(1+x) \cdot f_{(J / J)}(x) d x\right)^{2} \\
& =\frac{N}{J} \cdot \mathcal{S}^{2} \cdot \int_{0}^{\infty}\left(\log _{2}(1+x)\right)^{2} \cdot f_{(J / J)}(x) d x \\
& +\frac{N \cdot(N-1)}{J^{2}}\left(\mathcal{S} \int_{0}^{\infty} \log _{2}(1+x) f_{(J / J)}(x) d x\right)^{2} \\
& =\left\{\frac{N}{J} \cdot \mathcal{S}^{2} \cdot \int_{0}^{\infty}\left(\log _{2}(1+x)\right)^{2} \cdot f_{(J / J)}(x) d x\right\} \\
& +\left(\mathrm{E}\left[s_{j}\right]\right)^{2}-\frac{1}{N}\left(\mathrm{E}\left[s_{j}\right]\right)^{2} .
\end{aligned}
$$

Since we have already solved the second part of (13), we only have to focus on the first part:

$$
\begin{aligned}
\{\}= & \frac{N \mathcal{S}^{2}}{J} \cdot \sum_{i=0}^{J-1}\left(\begin{array}{c}
J-1 \\
i
\end{array}\right) \cdot(-1)^{i} \\
& \cdot \int_{0}^{\infty} \frac{J}{\bar{\gamma}}\left(\log _{2}(1+x)\right)^{2} \cdot e^{-\frac{x \cdot(i+1)}{\bar{\gamma}}} d x \\
= & N \mathcal{S}^{2} \cdot \sum_{i=0}^{J-1}\left(\begin{array}{c}
J-1 \\
i
\end{array}\right) \cdot(-1)^{i} \cdot \frac{e^{\frac{i+1}{\gamma}}}{\ln ^{2}(2)} \\
& \cdot\left(\frac{1}{(i+1)}\left[\left(C+\ln \left(\frac{i+1}{\bar{\gamma}}\right)\right)^{2}+\frac{\pi^{2}}{6}\right]\right. \\
& \left.-\frac{2}{\bar{\gamma}}{ }_{3} F_{3}\left(1,1,1 ; 2,2,2 ;-\frac{i+1}{\bar{\gamma}}\right)\right) .
\end{aligned}
$$

Based on Equations (14) and (12) we can obtain the second moment from Equation (13). This allows us then to compute the variance, from which we can finally compute the effective service capacity based on Equation (7).

\subsection{Effective Capacity of OFDMA with Link Adaptation per Terminal}

Next we consider the OFDMA system with link adaptation per terminal. Like in the previous case, all subcarriers are assigned to the terminal with the highest channel gain. However, due to the restrictions from link adaptation, we bound the modulation scheme of all subcarriers associated with a terminal to the weakest channel gain. Hence, the link adaption per terminal is driven by the lowest channel gain of the subcarriers assigned to a terminal. We are interested in quantifying the loss in comparison to an OFDMA system with adaptive modulation and with link adaptation. The 
PDF and CDF of the assigned subcarriers of an opportunistic OFDMA system with $J$ terminals are given by

$$
f_{(J / J)}(x)=\frac{J}{\bar{\gamma}} \cdot \sum_{i=0}^{J-1}\left(\begin{array}{c}
J-1 \\
i
\end{array}\right) \cdot(-1)^{i} \cdot e^{-\frac{x(i+1)}{\bar{\gamma}}} .
$$

and

$$
F_{(J / J)}(x)=\left(1-e^{-\frac{x}{\bar{\gamma}}}\right)^{J}
$$

Let $K$ denote the number of subcarriers that are assigned to a terminal. As we are interested in the distribution of the worst subcarrier SNR $\gamma^{(1 / K)(J)}$ out of $K$ i.i.d. distributed random SNRs which are assigned to a terminal, the resulting $\mathrm{CDF}$ and PDF of the lowest SNR is obtained as follows:

$$
\begin{aligned}
F_{(1 / K)(J)}(x) & =1-\left[1-F_{J / J}(x)\right]^{K} \\
& =1-\left[1-\left(1-e^{-\frac{x}{\bar{\gamma}}}\right)^{J}\right]^{K} .
\end{aligned}
$$

and

$$
\begin{aligned}
f_{(1 / K)(J)}(x)= & \frac{d}{d x} \cdot F_{(1 / K)(J)}(x) \\
= & \frac{K \cdot J}{\bar{\gamma}} \cdot\left[1-\left(1-e^{-\frac{x}{\bar{\gamma}}}\right)^{J}\right]^{K-1} \\
& \cdot\left(1-e^{-\frac{x}{\bar{\gamma}}}\right)^{J-1} \cdot e^{-\frac{x}{\bar{\gamma}}} \\
= & \frac{K \cdot J}{\bar{\gamma}} \cdot \sum_{i=0}^{K-1}\left(\begin{array}{c}
K-1 \\
i
\end{array}\right) \cdot(-1)^{i} \\
& \cdot \sum_{j=0}^{J i+J-1}\left(\begin{array}{c}
J i+J-1 \\
j
\end{array}\right) \cdot(-1)^{j} \cdot e^{-\frac{x \cdot(j+1)}{\bar{\gamma}(18)}}
\end{aligned}
$$

Now we can derive the first and second moment of the service process. For the mean, this results in:

$$
\begin{aligned}
& \mathrm{E}\left[s_{j}\right]=\sum_{k=1}^{N} \operatorname{Pr} .\left\{X_{j}=k\right\} \cdot k \cdot \mathrm{E}\left[s_{j}^{(1 / k)(J), 1}\right] \\
& =\sum_{k=1}^{N}\left(\begin{array}{l}
N \\
k
\end{array}\right) \cdot\left(\frac{1}{J}\right)^{k} \cdot\left(1-\frac{1}{J}\right)^{N-k} \cdot k \cdot \mathcal{S} \\
& \cdot \int_{0}^{\infty} \log _{2}(1+x) \cdot f_{(1 / k)(J)}(x) d x \\
& =\sum_{k=1}^{N}\left(\begin{array}{l}
N \\
k
\end{array}\right) \cdot\left(\frac{1}{J}\right)^{k} \cdot\left(1-\frac{1}{J}\right)^{N-k} \cdot k \cdot k \cdot J \\
& \cdot \sum_{i=0}^{k-1}\left(\begin{array}{c}
k-1 \\
i
\end{array}\right) \cdot(-1)^{i} \cdot \sum_{j=0}^{J i+J-1}\left(\begin{array}{c}
J i+J-1 \\
j
\end{array}\right) \\
& \cdot(-1)^{j} \cdot \int_{0}^{\infty} \frac{\mathcal{S}}{\bar{\gamma}} \cdot \log _{2}(1+x) \cdot e^{-\frac{x \cdot(j+1)}{\bar{\gamma}}} d x \\
& =\sum_{k=1}^{N}\left(\begin{array}{c}
N \\
k
\end{array}\right) \cdot\left(\frac{1}{J}\right)^{k} \cdot\left(1-\frac{1}{J}\right)^{N-k} \cdot k^{2} \cdot J \\
& \cdot \sum_{i=0}^{k-1}\left(\begin{array}{c}
k-1 \\
i
\end{array}\right) \cdot(-1)^{i} \cdot \sum_{j=0}^{J i+J-1}\left(\begin{array}{c}
J i+J-1 \\
j
\end{array}\right) \\
& \cdot(-1)^{j} \cdot \frac{\mathcal{S} \cdot e^{\frac{j+1}{\bar{\gamma}}}}{(j+1) \cdot \ln 2} \mathrm{E}_{1}\left(\frac{j+1}{\bar{\gamma}}\right) \text {. }
\end{aligned}
$$

For the second moment we obtain the following formula:

$$
\begin{aligned}
& \mathrm{E}\left[s_{j}^{2}\right]=\sum_{k=1}^{N} \operatorname{Pr} .\left\{X_{j}=k\right\} \\
& \cdot \mathrm{E}\left[\left(s_{j}^{(1 / k)(J), 1}+\cdots+s_{j}^{(1 / k)(J), k}\right)^{2}\right] \\
& =\sum_{k=1}^{N} \operatorname{Pr} \cdot\left\{X_{j}=k\right\} \cdot k^{2} \cdot \mathrm{E}\left[\left(s_{j}^{(1 / k)(J)}\right)^{2}\right] \\
& =\sum_{k=1}^{N}\left(\begin{array}{l}
N \\
k
\end{array}\right) \cdot\left(\frac{1}{J}\right)^{k} \cdot\left(1-\frac{1}{J}\right)^{N-k} \cdot k^{2} \cdot \mathcal{S}^{2} \\
& \cdot \int_{0}^{\infty}\left(\log _{2}(1+x)\right)^{2} \cdot f_{(1 / k)(J)}(x) d x \\
& =\sum_{k=1}^{N}\left(\begin{array}{l}
N \\
k
\end{array}\right) \cdot\left(\frac{1}{J}\right)^{k} \cdot\left(1-\frac{1}{J}\right)^{N-k} \cdot k^{2} \cdot k \cdot J \\
& \cdot \sum_{i=0}^{k-1}\left(\begin{array}{c}
k-1 \\
i
\end{array}\right) \cdot(-1)^{i} \cdot \sum_{j=0}^{J i+J-1}\left(\begin{array}{c}
J i+J-1 \\
j
\end{array}\right) \\
& \cdot(-1)^{j} \cdot \int_{0}^{\infty} \frac{\mathcal{S}^{2}}{\bar{\gamma}} \cdot\left(\log _{2}(1+x)\right)^{2} \cdot e^{-\frac{x \cdot(j+1)}{\bar{\gamma}}} d x \\
& =\sum_{k=1}^{N}\left(\begin{array}{l}
N \\
k
\end{array}\right) \cdot\left(\frac{1}{J}\right)^{k} \cdot\left(1-\frac{1}{J}\right)^{N-k} \cdot k^{3} \cdot J \\
& \cdot \sum_{i=0}^{k-1}\left(\begin{array}{c}
k-1 \\
i
\end{array}\right) \cdot(-1)^{i} \cdot \sum_{j=0}^{J i+J-1}\left(\begin{array}{c}
J i+J-1 \\
j
\end{array}\right) \\
& \cdot(-1)^{j} \cdot \frac{\mathcal{S}^{2}}{\bar{\gamma} \cdot \ln ^{2}(2)} \cdot e^{\frac{j+1}{\bar{\gamma}}} \\
& {\left[\frac{\left(C+\ln \left(\frac{j+1}{\bar{\gamma}}\right)\right)^{2} \cdot \bar{\gamma}}{j+1}+\frac{\pi^{2} \cdot \bar{\gamma}}{6(j+1)}\right.} \\
& \left.-2 \cdot{ }_{3} F_{3}\left(1,1,1 ; 2,2,2 ;-\frac{j+1}{\bar{\gamma}}\right)\right] .
\end{aligned}
$$

\subsection{Effective Capacity of OFDMA with Link Adaptation}

In the last case, the system applies again link adaptation. However, it is not applied individually per terminal but over all $N$ subcarriers (regardless of the assignments). Hence the deviation of the corresponding first and second moment is similar to the previous case, except that we have to substitute $K$ in the upper formulas by $N$. For convenience, we present below the corresponding expressions:

$$
\begin{aligned}
& \mathrm{E}\left[s_{j}\right]= N^{2} \cdot \mathcal{S} \cdot \sum_{i=0}^{N-1}\left(\begin{array}{c}
N-1 \\
i
\end{array}\right) \cdot(-1)^{i} \\
& \cdot \sum_{j=0}^{J \cdot i+J-1} \cdot\left(\begin{array}{c}
J i+J-1 \\
j
\end{array}\right) \cdot(-1)^{j} \\
& \frac{1}{(j+1) \cdot \ln (2)} \cdot e^{\frac{j+1}{\bar{\gamma}}} \cdot \mathrm{E}_{1}\left(\frac{j+1}{\bar{\gamma}}\right) \cdot(21) \\
& \mathrm{E}\left[s_{j}^{2}\right]=\left(N^{2}+\frac{N^{2}(N-1)}{J}\right) \cdot \sum_{i=0}^{N-1}\left(\begin{array}{c}
N-1 \\
i
\end{array}\right) \cdot(-1)^{i}
\end{aligned}
$$




$$
\begin{aligned}
& \cdot \sum_{j=0}^{J \cdot i+J-1}\left(\begin{array}{c}
J i+J-1 \\
j
\end{array}\right) \cdot(-1)^{j} \cdot \frac{\mathcal{S}^{2} \cdot e^{\frac{j+1}{\bar{\gamma}}}}{\bar{\gamma} \cdot \ln ^{2}(2)} \\
& \cdot\left(\frac{\bar{\gamma}}{j+1} \cdot\left[\left(C+\ln \left(\frac{j+1}{\bar{\gamma}}\right)\right)^{2}+\frac{\pi^{2}}{6}\right]\right. \\
& \left.-2 \cdot_{3} F_{3}\left(1,1,1 ; 2,2,2 ;-\frac{j+1}{\bar{\gamma}}\right)\right) .
\end{aligned}
$$

With the above formulas we are now able to compute the effective service capacity for all cases based on Equation (7).

\section{NUMERICAL ANALYSIS}

In this section we evaluate our analytical results numerically. The section is split into two parts. In the first subsection, we provide a validation of the various different analytical derivations we have presented previously. All our validation work is based on comparisons with simulation results. After validation we then turn to the investigation of the three different system approaches regarding queuing performance for various different parameter settings.

\subsection{Validation of Analysis}

The validation is actually performed in two steps. We first focus on validating the results for the mean and the variance of the corresponding service processes for the three different systems considered. Then, in a second step we validate the analytical derivation of the maximum sustainable rate as obtained by Equation (10) by simulating the corresponding queuing system and observing the delay violations in comparison to the analytical predictions.

The basic simulation set up considered consists of $J=5$ terminals and $N=48$ subcarriers for the three different system approaches. Each simulation was based on 800.000 frame iterations repeated in 30 independent runs (by controlling the seed for generating the random fading coefficients). As assumed for the analysis, for the fading coefficients we considered an exponential distribution with neither correlation in time nor in frequency. The frame length was set to $1 \mathrm{~ms}$ while each subcarrier featured 10 symbols per frame. As QoS targets we assumed an outage probability of $\mathcal{P}_{j}=0.03$ and a target delay of $d_{j}=30 \mathrm{~ms}$ for all system approaches. For each simulation run we then observed the number of frames for which this QoS target could not be fulfilled and averaged this observation over the 30 different runs over all terminals. We repeated this for four different values of the average SNR $\bar{\gamma}: 5,10,20$ and $30 \mathrm{~dB}$.

We start with presenting the validation results for the OFDMA system with adaptive modulation. In Figure 1 the validation results are shown. The figure shows actually three plots (as is the case for all other system validation results as well). The first plot compares the average and standard deviation of the service process as obtained by the presented analysis in Section 3.1 with the empirical mean and standard deviation as obtained from the simulation. Notice that for all considered SNR points the analysis matches the empirical values. The other two plots in Figure 1 show the empirical outage probability observed for different arrival rates to the system. For this, we first obtained analytically the maximum sustainable arrival rate to the OFDMA system according to the QoS targets as discussed above. For the four different SNR points considered, these rates are given in Table 1. Recall that these rates, as obtained by Equation (10),

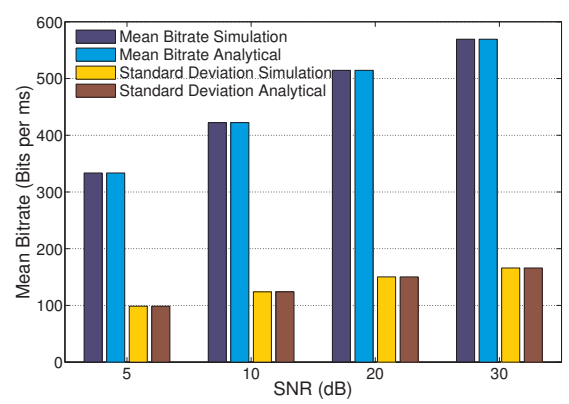

(a) Bitrate

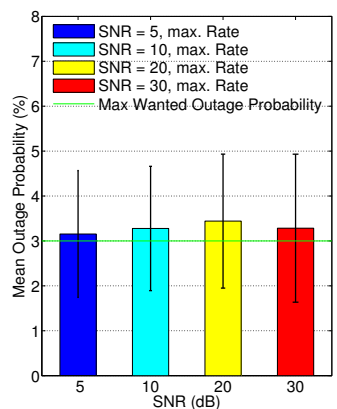

(b) Outages $r_{j}^{*}$

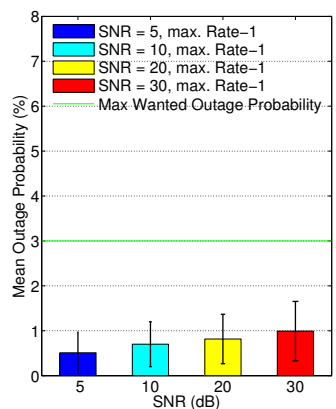

(c) Outages $r_{j}^{*}-1$
Figure 1: Mean and standard deviation of the analytically derived vs. the empirically observed bit rates as well as violation probabilities for OFDMA in combination with adaptive modulation. All figures show results for four different average SNR values of $5,10,20$ and $30 \mathrm{~dB}$.

\begin{tabular}{c|c|c|c|c} 
Scheme & AR 5dB & AR 10dB & AR 20dB & AR 30dB \\
\hline \hline LA & 180.61 & 255.54 & 339.39 & 391.13 \\
LA p.T & 228.78 & 309.84 & 397.46 & 450.57 \\
AM & 331.78 & 420.12 & 511.87 & 566.46
\end{tabular}

Table 1: Calculated maximum arrival rates (AR) in $\mathrm{bits} / \mathrm{ms}$ for the five different system set ups considered for different average SNR values and the QoS targets given by a delay of $30 \mathrm{~ms}$ and an outage probability of 0.03 .

should approximately fulfill the QoS-requirements. Hence, we first conducted simulations setting the arrival rate exactly to these analytical results. The corresponding empirical outage probabilities can be found in Figure 1b. Notice that for all four SNR points the empirically determined outage probabilities as observed during the simulations are close to the required outage probability of 0.03 but violate them slightly (which is - however - within the also considered confidence interval). By reducing the arrival rates from Table 1 by exactly $1 \mathrm{bit} /$ frame, the target outage probabilities were met as shown in the third plot of Figure 1. Note that a reduction of $1 \mathrm{bit} /$ frame equals a proportion of only $0.3 \%$ or less of the total rate. Hence, this validates the maximum sustainable rate as obtained by Equation (10) noting that it is rather an upper bound.

We find more or less the same results regarding the validation for the two link adaptation OFDMA systems in Figure 2 (regarding link adaptation over all subcarriers) and Figure 3 (regarding link adaptation per terminal). Note 
that the calculation of the binomial coefficients in determining the mean and variance of the OFDMA systems with link adaptation might lead to numerical instabilities due to the large numbers involved. Hence, in our evaluations we approximated the coefficients by applying Romberg's method [4]. Nevertheless, the simulations validate the application of this approximation as well (all analytical results presented in Figure 2 and Figure 3 have been obtained based on the Romberg method).

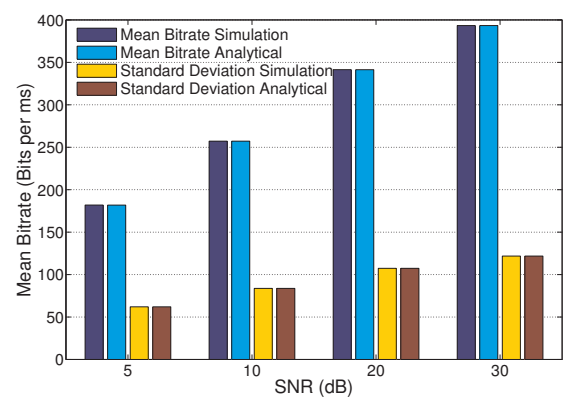

(a) Bitrate

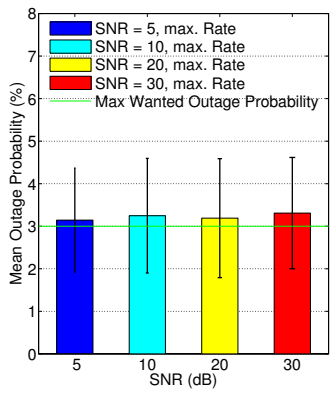

(b) Outages $r_{j}^{*}$

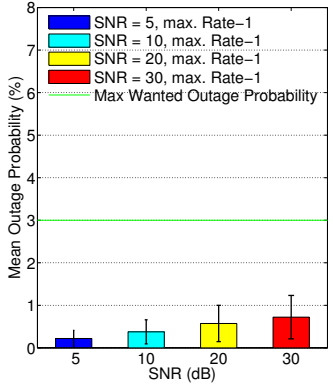

(c) Outages $r_{j}^{*}-1$
Figure 2: Mean and standard deviation of the analytically derived vs. the empirically observed bit rates as well as violation probabilities for OFDMA in combination with link adaptation. All figures show results for four different average SNR values of 5, 10, 20 and $30 \mathrm{~dB}$.

\subsection{Analysis of Sustainable Rates}

In this section we have a closer look at the queuing performance (i.e. analytically derived maximum sustainable rate) for the different system approaches. We investigated the following simulation set-up: We consider again a frame length of $1 \mathrm{~ms}$, but this time there can be 144 symbols transmitted per frame per subcarrier with a total amount of 48 subcarriers. This equals a set up of an LTE network with $10 \mathrm{MHz}$ bandwidth, which has 48 resource blocks, each with 144 symbols per frame. We considered for this setting a varying number of terminals in the cell which increased from 1 up to the point where no positive (bit) rate could be supported any longer. Furthermore, we considered four different pairs of QoS parameters consisting of target delay $d_{j}$ and outage probability $\mathcal{P}_{j}: d_{j}=100 \mathrm{~ms}$ and $\mathcal{P}_{j}=0.1$ (solid lines in the figures); $d_{j}=75 \mathrm{~ms}$ and $\mathcal{P}_{j}=0.07$ (dashed/dotted lines); $d_{j}=50 \mathrm{~ms}$ and $\mathcal{P}_{j}=0.03$ (dashed lines with marker + ); $d_{j}=25 \mathrm{~ms}$ and $\mathcal{P}_{j}=0.01$ (purely dashed lines). For the different system approaches and scenarios we then calculated

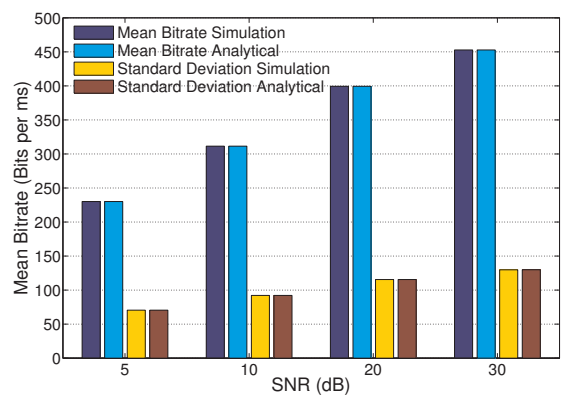

(a) Bitrate

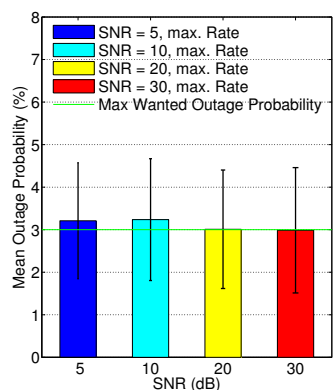

(b) Outages $r_{j}^{*}$

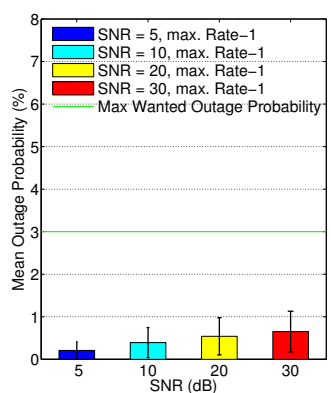

(c) Outages $r_{j}^{*}-1$
Figure 3: Mean and standard deviation of the analytically derived vs. the empirically observed bit rates as well as violation probabilities for OFDMA in combination with link adaptation per terminal. All figures show results for four different average SNR values of 5, 10, 20 and $30 \mathrm{~dB}$.

two performance metrics: For one the maximum sustainable rate per terminal while we also considered the aggregated (total) rate per cell (maximum sustainable rates multiplied with the number of terminals per cell). This was performed for two different settings of the average SNR: 10 and $20 \mathrm{~dB}$. Figure 4 displays the legend used in the following figures for the results of the different set-ups.

\begin{tabular}{|c|c|}
\hline 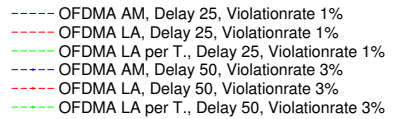 & $\begin{array}{c}\text { OFDMA AM, Delay } 75 \text {, Violationrate } 7 \% \\
\text { OFDMA LA, Delay } 75, \text { Violationrate } 7 \% \\
\text { OFDMA LA per T., Delay } 75 \text {, Violationrate } 7 \% \\
\text { OFDMA AM, Delay } 100 \text {, Violationrate } 10 \% \\
\text { OFDMA LA, Delay } 100, \text { Violationrate } 10 \% \\
\text { OFDMA LA per T., Delay 100, Violationrate } 10 \%\end{array}$ \\
\hline
\end{tabular}

Figure 4: Caption for the figures dealing with the maximum sustainable rates (Figures 5, 6, 7 and 8). The different system approaches are distinguished by color while the different QoS-requirements are distinguished by the line appearance.

Figures 5 and 6 show the resulting graphs for the different modulation schemes and an average SNR of $20 \mathrm{~dB}$. With a growing number of terminals, the differences between adaptive modulation and link adaptation per terminal become smaller since there are less subcarriers per terminal available and therefore the positive effect of adaptive modulation is weakened. With decreasing QoS-requirements, i.e. larger delays and bigger outage probabilities, the maximum throughput per cell increases as well as the number of ter- 
minals that lead to the maximum throughput. This is clear, since for strict QoS-requirements we need some resources to guarantee the delay bounds, whereas for relaxed requirements we can buffer the data longer and therefore support a larger data rate per terminal. As an overview, Table 2 shows the optimal number of terminals in terms of the total throughput per cell. Also, it lists in brackets the maximum sustainable rate per terminal leading to this throughput.

\begin{tabular}{l||l|l||l|l||lll}
\multicolumn{1}{c||}{} & \multicolumn{2}{c||}{ AM } & \multicolumn{2}{c||}{ LApT } & \multicolumn{2}{c}{ LA } \\
\hline avgSNR & $20 \mathrm{~dB}$ & $10 \mathrm{~dB}$ & $20 \mathrm{~dB}$ & $10 \mathrm{~dB}$ & $20 \mathrm{~dB}$ & $10 \mathrm{~dB}$ \\
\hline$d_{j} 25 \mathrm{~ms}$ & 19 & 21 & 26 & 28 & 29 & 31 \\
$P_{j} 1 \%$ & 1302 & 989.4 & 917.5 & 714.3 & 729.3 & 560.7 \\
\hline$d_{j} 50 \mathrm{~ms}$ & 41 & 45 & 50 & 55 & 58 & 64 \\
$P_{j} 3 \%$ & 639.8 & 493.4 & 515.8 & 396.7 & 401.1 & 302.8 \\
\hline$d_{j} 75 \mathrm{~ms}$ & 70 & 78 & 82 & 90 & 99 & 109 \\
$P_{j} 7 \%$ & 387.8 & 295.9 & 327.9 & 254.0 & 247.7 & 188.7 \\
\hline$d_{j} 100 \mathrm{~ms}$ & 100 & 111 & 113 & 124 & 138 & 153 \\
$P_{j} 10 \%$ & 276.8 & 212.6 & 243.3 & 189.0 & 182.9 & 138.9
\end{tabular}

Table 2: Displayed are the numbers of terminals that lead to the best cell throughput for the OFDMA system approaches with an average SNR of 20 and $10 \mathrm{~dB}$ respectively (first lines). The number in second lines represents the maximum sustainable rate per terminal in bit/ms that lead to the maximum.

Considering the maximum sustainable rates per terminal (Figure 6) we can see, that the differences between the various QoS-requirements lead to only small changes of the rates compared to the total amount per terminal. The zoomed part shows, that the biggest difference occurs between a delay of $25 \mathrm{~ms}$ with $1 \%$ outage probability and a delay of $50 \mathrm{~ms}$ with an outage probability of $3 \%$. Here the difference between the data rates is about $50 \mathrm{bit} / \mathrm{ms}$, whereas the differences between the more relaxed ones is in the range of 4 to $10 \mathrm{bits} / \mathrm{ms}$. The peak data rate per terminal for the link adaptation systems is reached at a total number of two terminals in the cell. This happens, since for two terminals the possibility for a better lowest subcarrier SNR is higher than the possibility considering the case with only one terminal and hence the used modulation scheme which depends on the SNR of the worst subcarrier is better. With a growing number of terminals the losses of subcarriers is more important for the terminals than the better modulation scheme and therefore the data rate per terminal decreases.

Considering the results for an average SNR of $10 \mathrm{~dB}$ in Figure 7 indicate, that with a decreasing received signal strength the number of terminals that lead to a maximum aggregated rate per cell increases. This trend also continues for lower average SNRs (not displayed in this paper). While the optimal number of terminals for OFDMA with adaptive modulation and $20 \mathrm{~dB}$ was 19 terminals in case of a delay of $25 \mathrm{~ms}$, it is 21 terminals for $10 \mathrm{~dB}$ (compare Table 2).

Hence, with a lower average SNR we need more terminals to get an optimal cell throughput. This indicates that for lower SNR values the effect of the multiuser diversity is more important for a high data rate per cell than for higher SNR values. The effect is even stronger for the relaxed QoSrequirements as shown in Figure 8 . The optimal number of terminals in terms of a high data rate per terminal is reached for a total number of 2 or 3 terminals per cell in case of link adaptation and link adaptation per terminal, whereas for higher average SNR values this optimum was reached at only 2 terminals per cell. With a decreasing average SNR

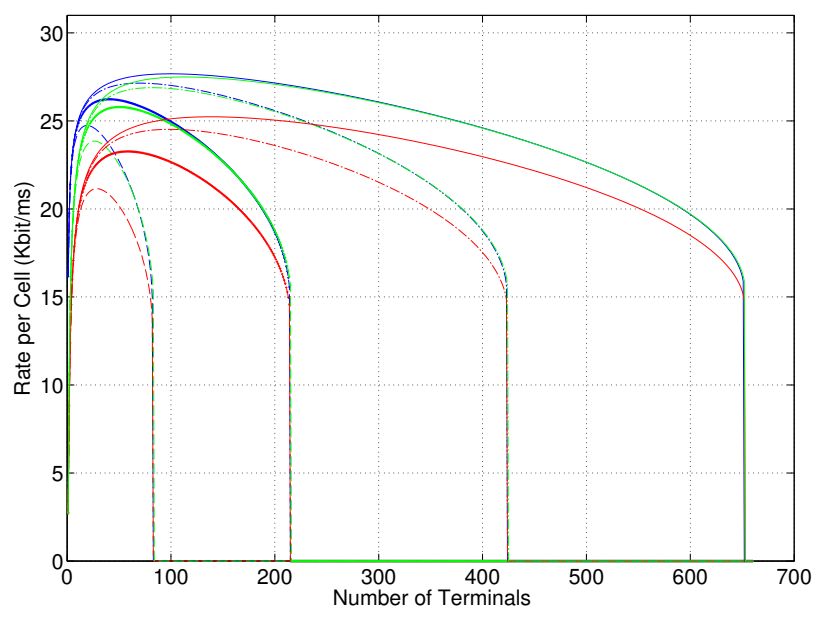

Figure 5: Aggregated bit rate per cell with an average SNR of $20 \mathrm{~dB}$.

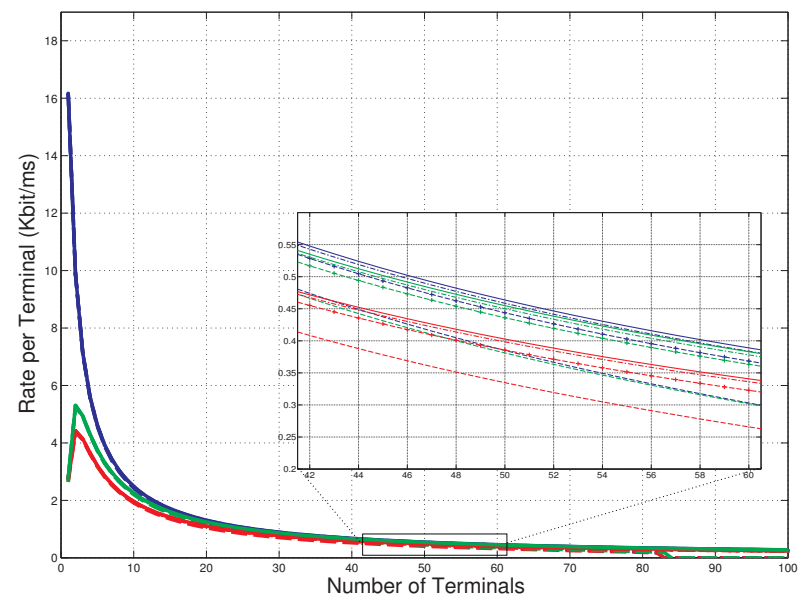

Figure 6: Maximum sustainable rate per terminal per ms with an average SNR of $20 \mathrm{~dB}$.

the total throughput per cell also decreases, but the number of terminals, for which we can not calculate a maximum sustainable rate for given QoS-requirements stays nearly the same.

The differences between OFDMA with adaptive modulation and OFDMA with link adaptation per terminal are only minimal in terms of sustainable rates per cell and sustainable rate per terminal, whereas the differences between these schemes and OFDMA with link adaptation are much bigger with about $50 \mathrm{bits} / \mathrm{ms}$ for all QoS-requirements.

\section{CONCLUSIONS}

With the expected rise of video applications in upcoming cellular networks of the fourth generation, analytical quality of service models for such networks become important. In this paper we provide approximations for the queuing delay of opportunistic OFDMA networks based on the effective service capacity framework. These approximations can serve for example for admission control in such systems later on. Our analysis covers three distinct cases for adapting the modulation type to the channel states (once the subcarriers have been assigned): Adaptive modulation per subcarrier, 


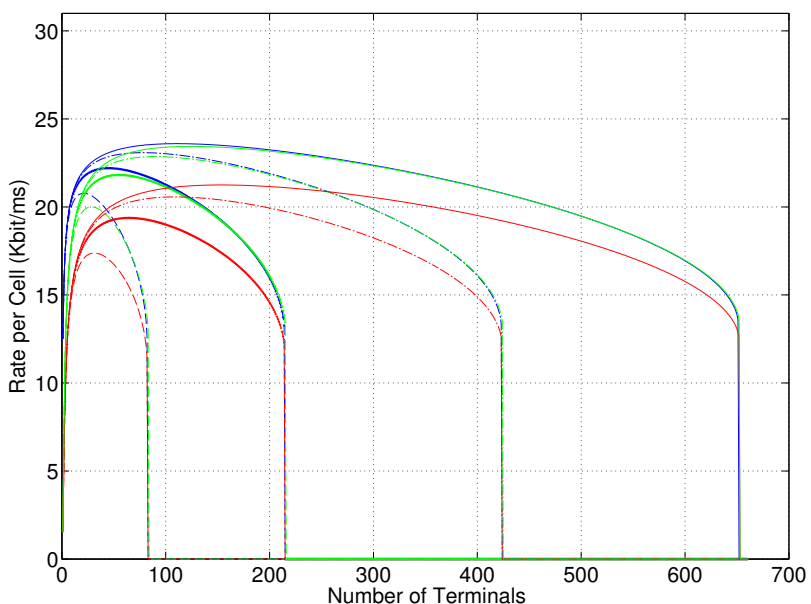

Figure 7: Aggregated bit rate per cell with an average SNR of $10 \mathrm{~dB}$.

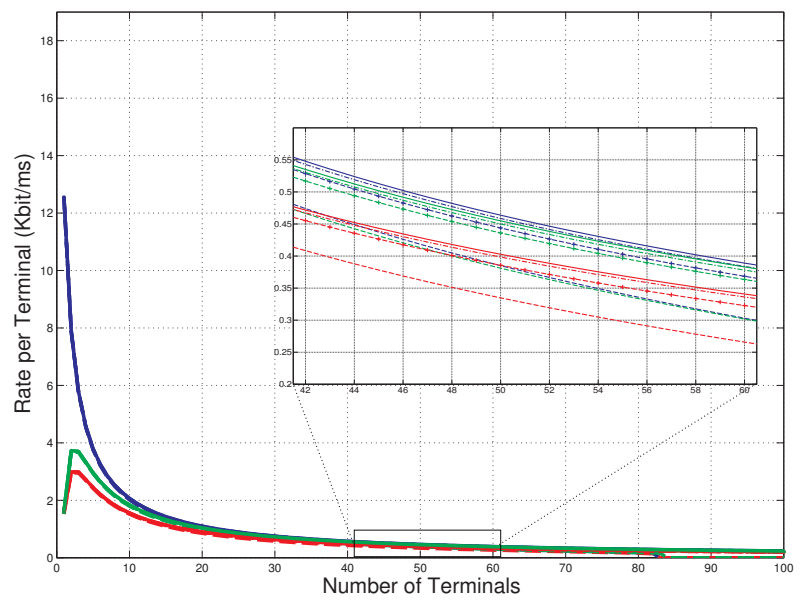

Figure 8: Maximum sustainable rate per terminal per ms with an average SNR of $10 \mathrm{~dB}$.

link adaptation per terminal as well as link adaptation over all subcarriers. Interestingly, we find that adaptive modulation and link adaptation per terminal provide an almost identical performance. In general, all OFDMA systems feature an optimal number of terminals to be present in the cell which maximizes the joint (QoS-constrained) traffic rate that can be forwarded in the down-link. However, different system set-ups feature different optima. For future work, we will extend our work to interference-limited systems.

\section{REFERENCES}

[1] M. Bohge, J. Gross, M. Meyer, and A. Wolisz. Dynamic Resource Allocation in OFDM Systems: An Overview of Cross-Layer Optimization Principles and Techniques. IEEE Network, 21(1):53-59, 2007.

[2] C. Chang. Stability, queue length and delay of deterministic and stochastic queueing networks. IEEE Trans. Automat. Contr., 39(5):913-931, 1994.

[3] E. Dahlmann, S. Parkvall, J. Sköld, and P. Beming. $3 G$ Evolution: HSPA and LTE for Mobile Broadband. Academic Press, 2008.
[4] R. W. Freund and R. H. W. Hoppe. Stoer/Bulirsch: Numerische Mathematik 1. Springer-Verlag Berlin Heidelberg, 2007.

[5] J. Gross. Admission control based on OFDMA channel transformations. In Proc. of 10th IEEE International Symposium on a World of Wireless, Mobile and Multimedia Networks (WoWMoM), June 2009.

[6] J. Gross. Scheduling with outdated csi: Effective service capacities of optimistic vs. pessimistic policies. In Proc. of the IEEE International Workshop on Quality-of-Service (IWQoS), Coimbra, Portugal, June 2012.

[7] T. Jia and Z. Xi. QoS-Driven Adaptive Power and Rate Allocation for Multichannel Communications in Mobile Wireless Networks. In Proc. of the IEEE International Symposium on Information Theory (ISIT), Seattle, USA, July 2006.

[8] A. Kumar, D. Manjunath, and J. Kuri. Communication Networking: An Analytical Approach. Morgan Kaufmann, 2004.

[9] F. Naghibi and J. Gross. How bad is interference in ieee 802.16e systems? In Proc. of the 16th European Wireless Conference (EW'10), April 2010.

[10] D. Niyato and E. Hossain. Adaptive Fair Subcarrier/Rate Allocaiton in Multirate OFDMA Networks: Radio Link Level Queueing Performance Analysis. IEEE Trans. Veh. Technol., 55(6):1897-1907, 2006.

[11] S. Shakkottai. Effective capacity and qos for wireless scheduling. IEEE Trans. Automat. Contr., 53(3):749 $-761,2008$.

[12] H. Song, R. Kwan, and J. Zhang. General results on snr statistics involving eesm-based frequency selective feedbacks. IEEE Trans. Wireless Commun., 9(5):1790 $-1798,2010$.

[13] B. Soret, M. Aguayo-Torres, and J. Entrambasaguas. Multiuser capacity for heterogeneous QoS constraints in uncorrelated Rayleigh channels. In Proc. of IEEE Information Theory Workshop (ITW), pages 278-282, Oct. 2009.

[14] B. Soret, M. C. Aguayo-Torres, and J. T. Entrambasaguas. Capacity with Explicit Delay Guarantees for Generic Sources over Correlated Rayleigh Channel. IEEE Trans. Wireless Commun., 9(6):1901-1911, 2010.

[15] D. Wu and R. Negi. Effective capacity: a wireless link model for support of quality of service. IEEE Trans. Wireless Commun., 2(4):630-643, 2003.

[16] D. Wu and R. Negi. Downlink scheduling in a cellular network for quality-of-service assurance. IEEE Trans. Veh. Technol., 53(5):1547-1557, 2004.

[17] D. Wu and R. Negi. Effective capacity channel model for frequency-selective fading channels. In Proc. of the ICST Second International Conference on Quality of Service in Heterogeneous Wired/Wireless Networks, Orlando, USA, Aug. 2005.

[18] D. Wu and R. Negi. Utilizing multiuser diversity for efficient support of quality of service over a fading channel. IEEE Trans. Veh. Technol., 54(3):1198-1206, 2005 . 